\title{
Development of three-dimensional printing polymer-ceramic scaffolds with enhanced compressive properties and tuneable resorption
}

\author{
Zuoxin Zhou ${ }^{\mathrm{a}}$, Eoin Cunningham ${ }^{\mathrm{b}}$, Alex Lennon ${ }^{\mathrm{b}}$, Helen McCarthy ${ }^{\mathrm{d}}$, Fraser Buchanan ${ }^{\mathrm{b}}$, Nicholas Dunne, ${ }^{\mathrm{c}, \mathrm{d}, \mathrm{e}, \mathrm{f}, *}$ \\ a Centre for Additive Manufacturing, Faculty of Engineering, The University of Nottingham, UK \\ ${ }^{\mathrm{b}}$ School of Mechanical and Aerospace Engineering, Queen's University Belfast, UK \\ ${ }^{c}$ School of Mechanical and Manufacturing Engineering, Dublin City University, Dublin 9, Ireland \\ ${ }^{d}$ Centre for Medical Engineering Research, School of Mechanical and Manufacturing Engineering, Dublin City University, Dublin 9, Ireland \\ e School of Pharmacy, Queen's University Belfast, UK \\ f Trinity Centre for Bioengineering, Trinity Biomedical Sciences Institute, Trinity College Dublin, Dublin 2, Ireland
}

\section{A R T I C L E I N F O}

\section{Keywords:}

Additive manufacturing

3D printing

Hydroxyapatite

Calcium sulphate

Poly( $\varepsilon$-caprolactone)

Tissue-engineering bone scaffold

Dynamic resorption

\begin{abstract}
A B S T R A C T
In this study, bone tissue engineered scaffolds fabricated via powder-based 3D printing from hydroxyapatite (HA) and calcium sulphate $\left(\mathrm{CaSO}_{4}\right)$ powders were investigated. The combination of using a fast resorbing $\mathrm{CaSO}_{4}$ based powder and the relatively slower HA powder represents a promising prospect for tuning the bioresorption of 3D printed (3DP) scaffolds. These properties could then be tailored to coincide with tissue growth rate for different surgical procedures. The manufactured scaffolds were infiltrated with poly( $\varepsilon$-caprolactone) (PCL). The PCL infiltrated the inter-particle spacing within the 3DP structures due to the nature of a loosely-packed powder bed and also covered the surface of ceramic-based scaffolds. Consequently, the average compressive strength, compressive modulus and toughness increased by $314 \%, 465 \%$ and $867 \%$, respectively. The resorption behaviour of the 3DP scaffolds was characterised in vitro using a high-throughput system that mimicked the physiological environment and dynamic flow conditions relevant to the human body. A rapid release of $\mathrm{CaSO}_{4}$ between Day 0 and 28 was commensurate with a reduction in scaffold mass and compressive properties, as well as an increase in medium absorption. In spite of this, HA particles, connected by PCL fibrils, remained within the microstructure after 56 days resorption under dynamic conditions. Consequently, a high level of structural integrity was maintained within the 3DP scaffold. This study presented a porous PCL-HA-CaSO ${ }_{4} 3 \mathrm{DP}$ structure with the potential to encourage new tissue growth during the initial stages of implantation and also offering sufficient structural and mechanical support during the bone healing phase.
\end{abstract}

\section{Introduction}

Additive Manufacturing (AM) has been highly recognised as a promising tool to fabricate patient-specific bone substitutes for the replacement and restoration of lost or irreparable bone tissues due to its unique ability to fully control the complex external shape and internal porous network for the printed scaffold. A virtual scaffold design can be directly generated from the computed tomography (CT) or magnetic resonance imaging (MRI) data for an individual patient. A precise construction of a three-dimensional (3D) structure allows prediction and monitoring of biomechanical behaviour of the scaffold and ensures that it provides sufficient support to the surrounding tissues and dynamic resorption to match the rate of newly-formed tissue ingrowth. Such de- velopments are crucial for the future of the interdisciplinary field of bone tissue engineering.

Powder-based inkjet 3D printing is an AM technology that builds a pre-defined structure via depositing binder droplets selectively on powder layers. The selection of powder and binder formulation is the key to a successful 3D printing process as it determines the green strength and structural integrity of a 3D printed (3DP) structure, as well as the reliability of the entire printing system. The $3 \mathrm{D}$ printing processability of calcium phosphate (CaP), which is the inorganic constituent of natural bone, has been investigated by introducing different binding approaches. Previous attempts have formulated acidic binders to initiate setting reactions between $\mathrm{CaP}$ and acids, or polymeric binders to glue CaP powder via polymer precipitation [1-5]. However, proprietary 3D printing systems are typically equipped with a thermally activated

\footnotetext{
* Corresponding author at: Centre for Medical Engineering Research, School of Mechanical and Manufacturing Engineering, Dublin City University, Dublin 9, Ireland.

Email address: nicholas.dunne@dcu.ie (N. Dunne)
} 

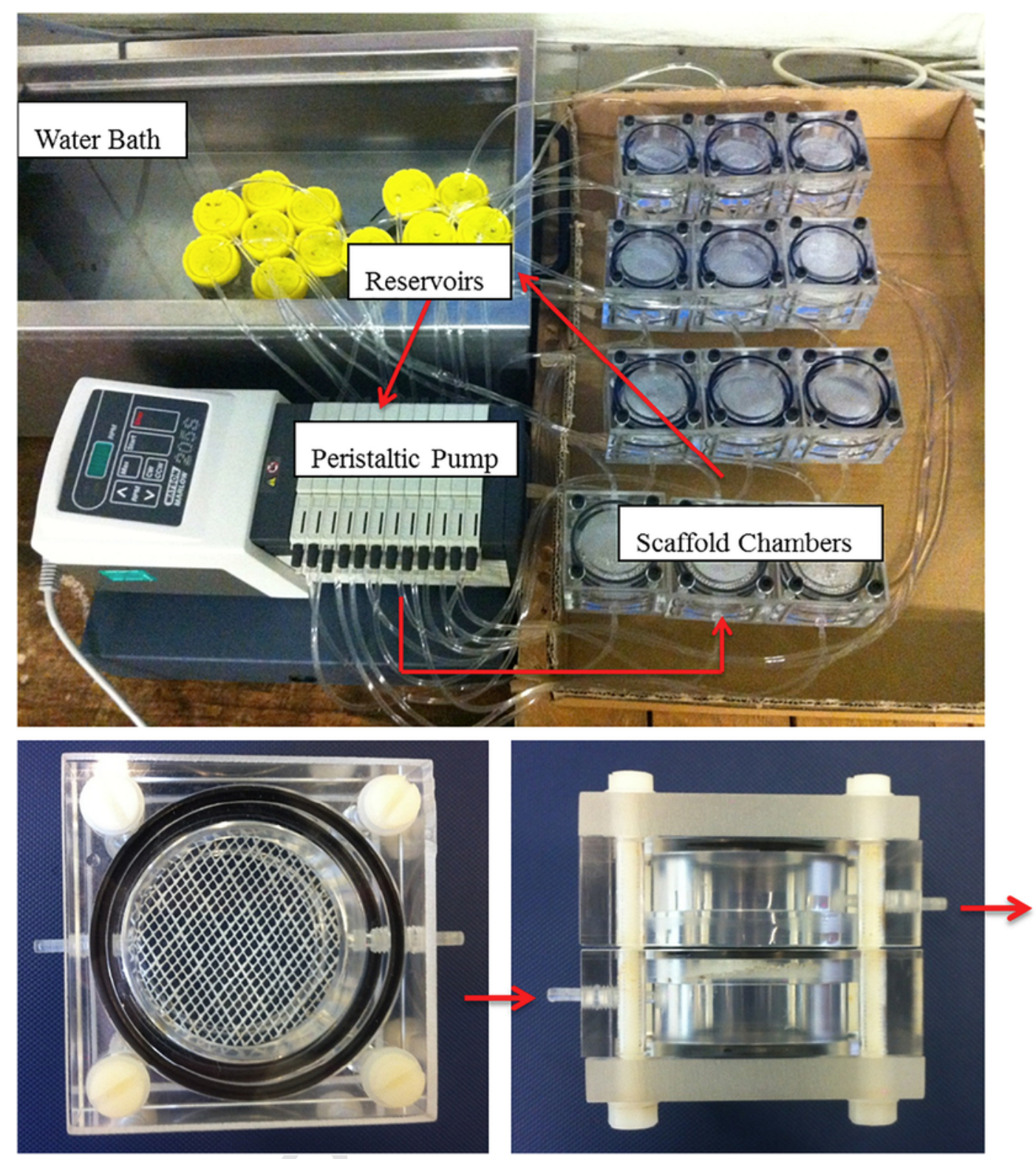

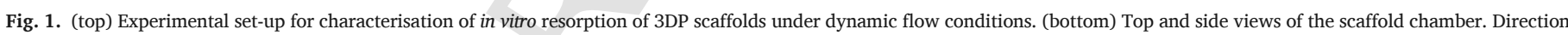
of fluid flow is indicated by the red arrows. (For interpretation of the references to colour in this figure legend, the reader is referred to the web version of this article.)

print-head, which only works reliably when water-based inks are used $[6,7]$. The use of acidic or polymeric binders can significantly compromise the working life and performance of these thermally activated print-heads. To eliminate the use of incompatible binders, some studies have incorporated in-bed binding adhesives into $\mathrm{CaP}$ powder bed designed to achieve high reactivity between the powder mixture and the standard water-based binder. Typical binding adhesives that have been used include starch [8], maltodextrin [9], and polyvinyl alcohol $[10,11]$. However, removal of these binding adhesives during the post-processing stage has resulted in relatively high levels of shrinkage $(18-20 \%)[1,12]$. A previous study proposed using a highly 3D printable bioceramic material, calcium sulphate $\left(\mathrm{CaSO}_{4}\right)$, and a binding adhesive for CaP powder [7]. A setting reaction occurred between $\mathrm{CaSO}_{4}$ and water-based binder, which solidified surrounding powders and, thus, offered high green strength for the printed structure.

Both $\mathrm{CaP}$ and $\mathrm{CaSO}_{4}$ can be resorbed by the host through chemical dissolution and osteoclast resorption processes [13]. However, these two materials have different resorption rates. The resorption of $\mathrm{CaSO}_{4}$ occurs during the early stages of implantation, often within a month $[14,15]$. Resorption of CaP occurs at a slower rate than $\mathrm{CaSO}_{4}[16]$. The most stable form of $\mathrm{CaP}$, hydroxyapatite (HA), demonstrates a resorption period of 12-36 months [17]. Ideally, the resorption rate required for the complete repair of a bone defect should coincide with the natural healing rate [18]. The challenge is that most biomaterials do not solely exhibit a resorption rate that matches the typical rate of bone formation [19]. The concept of combining $\mathrm{CaSO}_{4}$ and $\mathrm{CaP}$ to balance the resorption rate has been proposed in many studies [20-22]. It has been reported that the $\mathrm{CaP}-\mathrm{CaSO}_{4}$ composite exhibited a resorption rate closely matching the rate of new bone formation [19]. Here the rapid dissolution of $\mathrm{CaSO}_{4}$ during the early stages of implantation created porous spaces favouring cell activity, while HA remained and acted as a support for newly formed tissue $[23,24]$. The incorporation of $\mathrm{HA}$ into $\mathrm{CaSO}_{4}$ could also enhance osteoconductivity due to its crystalline structure and mineral apatite components analogous to natural bone $[19,25]$. 


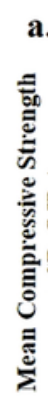

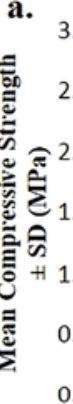
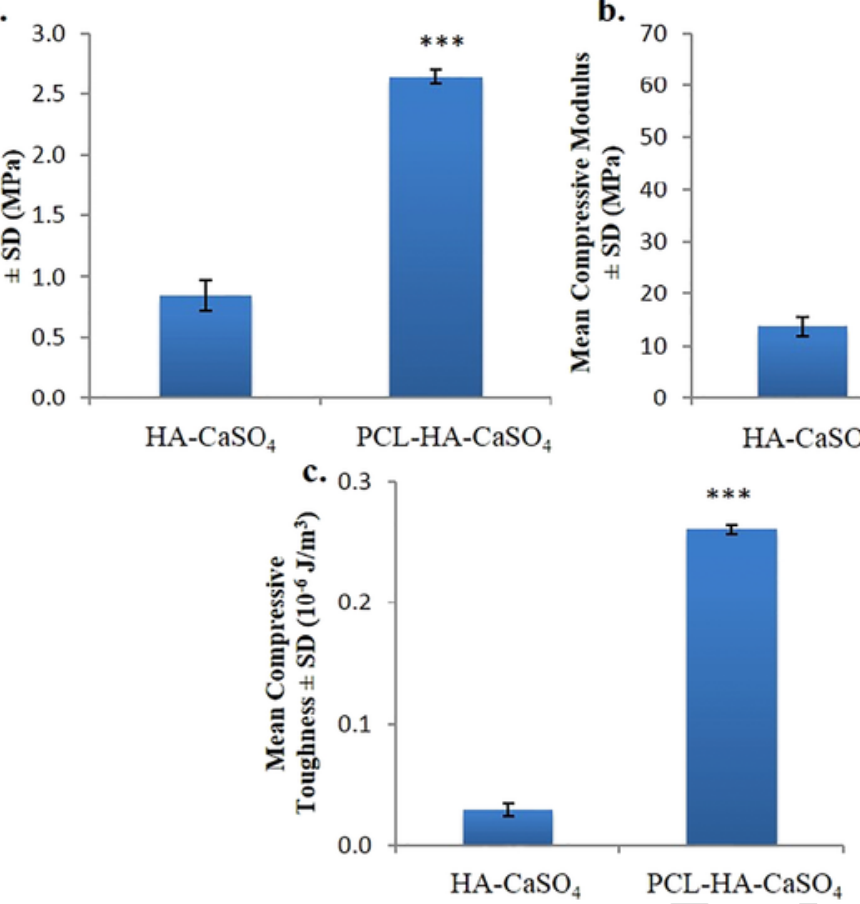

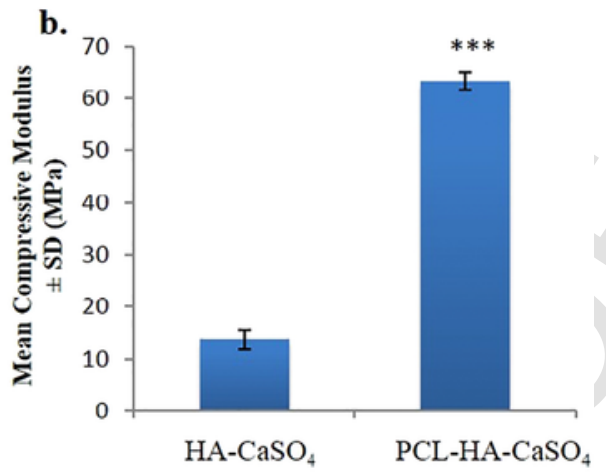

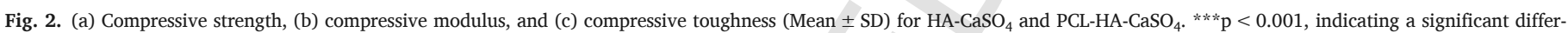
ence between $\mathrm{HA}_{-} \mathrm{CaSO}_{4}$ and PCL-HA-CaSO ${ }_{4}$.
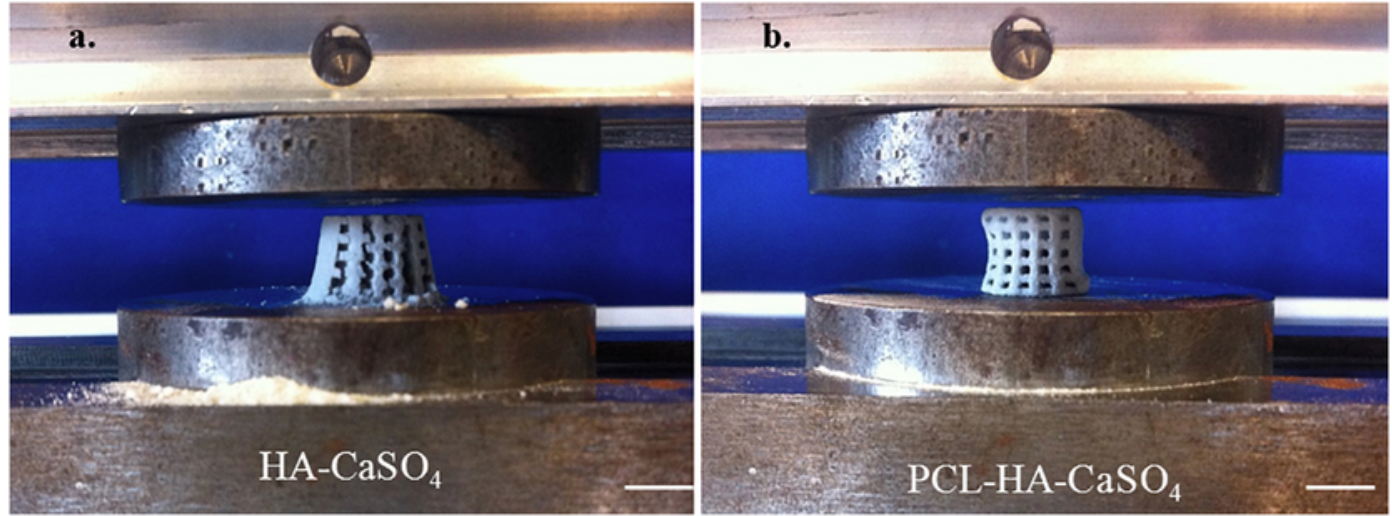

Fig. 3. Typical compressive deformation for $\mathrm{HA}^{-\mathrm{CaSO}_{4}}$ and $\mathrm{PCL}-\mathrm{HA}-\mathrm{CaSO}_{4} 3 \mathrm{DP}$ scaffolds and difference in scaffold integrity post-failure. Scale bar $=10 \mathrm{~mm}$.

In this study, bioceramic scaffolds were $3 \mathrm{DP}$ from a $\mathrm{HA}-\mathrm{CaSO}_{4}$ powder formulation. The printed scaffolds were post-treated using poly( $\varepsilon$-caprolactone) (PCL) to infiltrate the inter-particle spacing; PCL infiltration was demonstrated as a highly efficient method to improve the overall performance of 3DP bioceramic scaffolds [26]. The compressive properties, microstructural morphologies and in vitro resorption behaviour, under dynamic flow conditions, of 3DP composite scaffolds were subsequently investigated.

\section{Materials and methods}

\subsection{Materials}

The combination of $25 \mathrm{wt} \%$ HA (Capital ${ }^{\circledR}$, Plasma Biotal Ltd., UK) and $75 \mathrm{wt} \%$ hemihydrate $\mathrm{CaSO}_{4}$ powder (ZP102, Z Corporation, UK) was used as the powdered material for 3DP manufacturing. The HA powder was milled using a planetary mill (Pulverisette 6, Fristch $\mathrm{GmbH}$, Germany) and then sieved so as to achieve a particle size distribution similar to the hemihydrate $\mathrm{CaSO}_{4}$ powder, which had $\mathrm{D}_{10}, \mathrm{D}_{50}$, and $\mathrm{D}_{90}$ equal to $45.68 \mu \mathrm{m}, 65.35 \mu \mathrm{m}$, and $94.40 \mu \mathrm{m}$, as measured by laser diffraction using a two laser Sympatec HELOS/BF Particle Sizer (Sympatec Ltd., UK). Detailed powder preparation procedures were reported in a previous study [7]. Standard water-based binder (ZB 7, Z Corporation, UK) was used as the binder material. PCL powder (Capa 6506, Perstorp Ltd., UK) was dissolved (12\% w/w) in chloroform (288306, Sigma-Aldrich, UK) using an ADS-HP1 hotplate stirrer (Asynt Ltd., UK) at 3000 RPM until complete dissolution. Tris-HCl buffer solution $(\mathrm{pH}=7.4)$ was used as the physiological medium during the in vitro resorption test.

\subsection{Manufacturing process}

A cylindrical porous scaffold (diameter and height $=13.2 \mathrm{~mm}$, pore and strut size $=1.2 \mathrm{~mm}$ ) was designed and manufactured from the HA-CaSO ${ }_{4}$ powder using a ZCorp 310 3D printer (Z Corporation, US). The design of an interconnected porous structure can vary greatly according to its overall porosity and the size, shape and arrangement of the pores. Ultimately the ideal design should meet geometrical and me- 

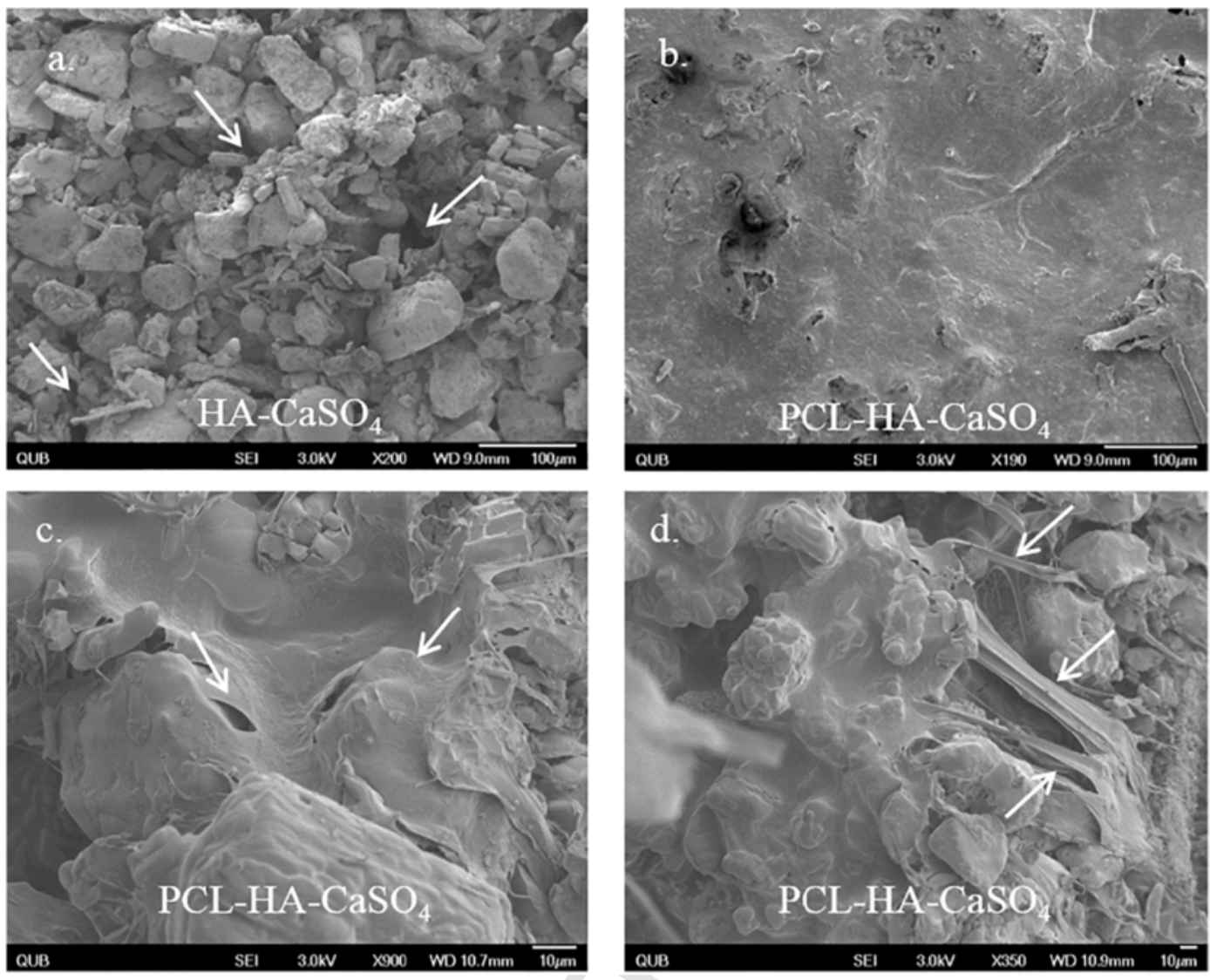

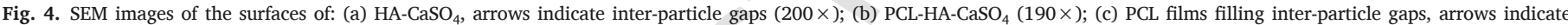

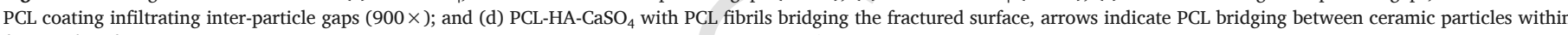
fractured surface $(350 \times)$.

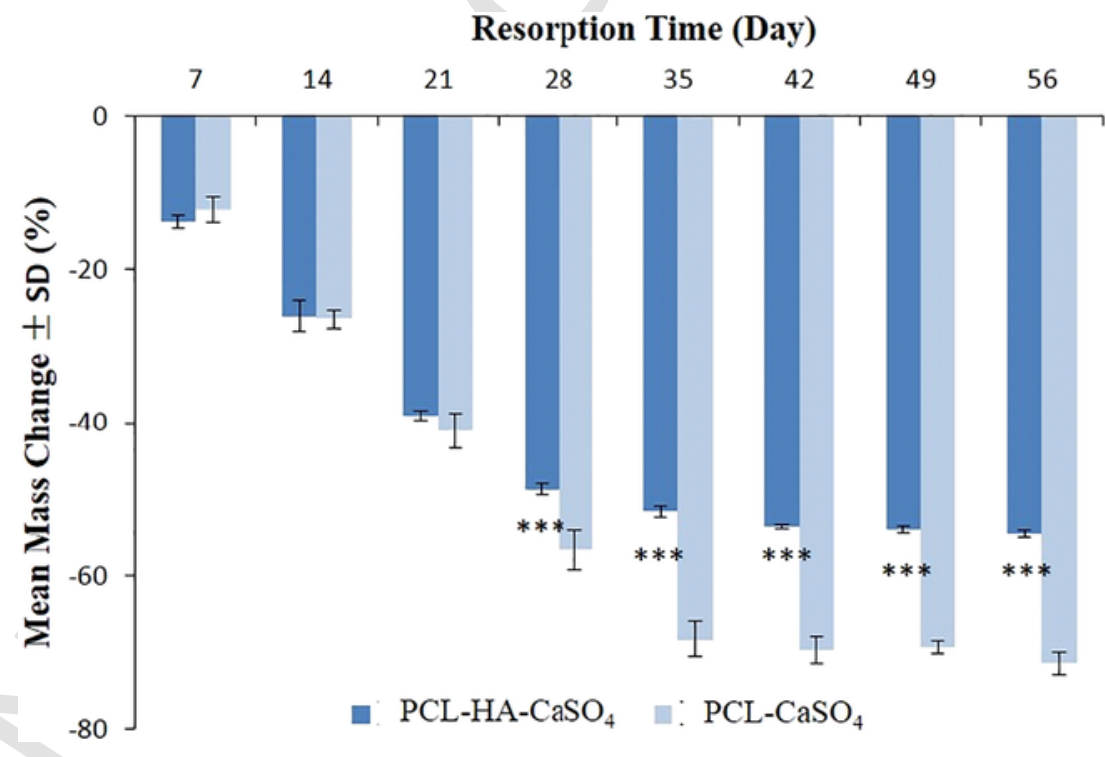

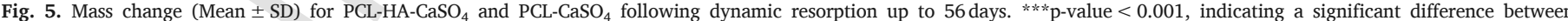
PCL-HA-CaSO ${ }_{4}$ and PCL-CaSO ${ }_{4}$ at the same time point.

chanical requirement towards a specific bone tissue engineering application in order facilitate effective bone healing. Nevertheless for materials research purposes, a cylindrical shaped specimen of regular porosity and pore architecture was chosen for this study as it have been previously used in a successful manner [26]. During the 3DP process, each binder droplet was selectively deposited on the print bed to build a cross-sectional layer of the structure. The overall structure was 3D-printed layer by layer consecutively - with a layer thickness of $100 \mu \mathrm{m}$. After the 3D printing process, the unbound powder was removed using compressed air.

Each 3DP scaffold was fully immersed in the PCL solution for $30 \mathrm{~s}$ to obtain PCL infiltration. Thereafter, the 3DP scaffold was removed 


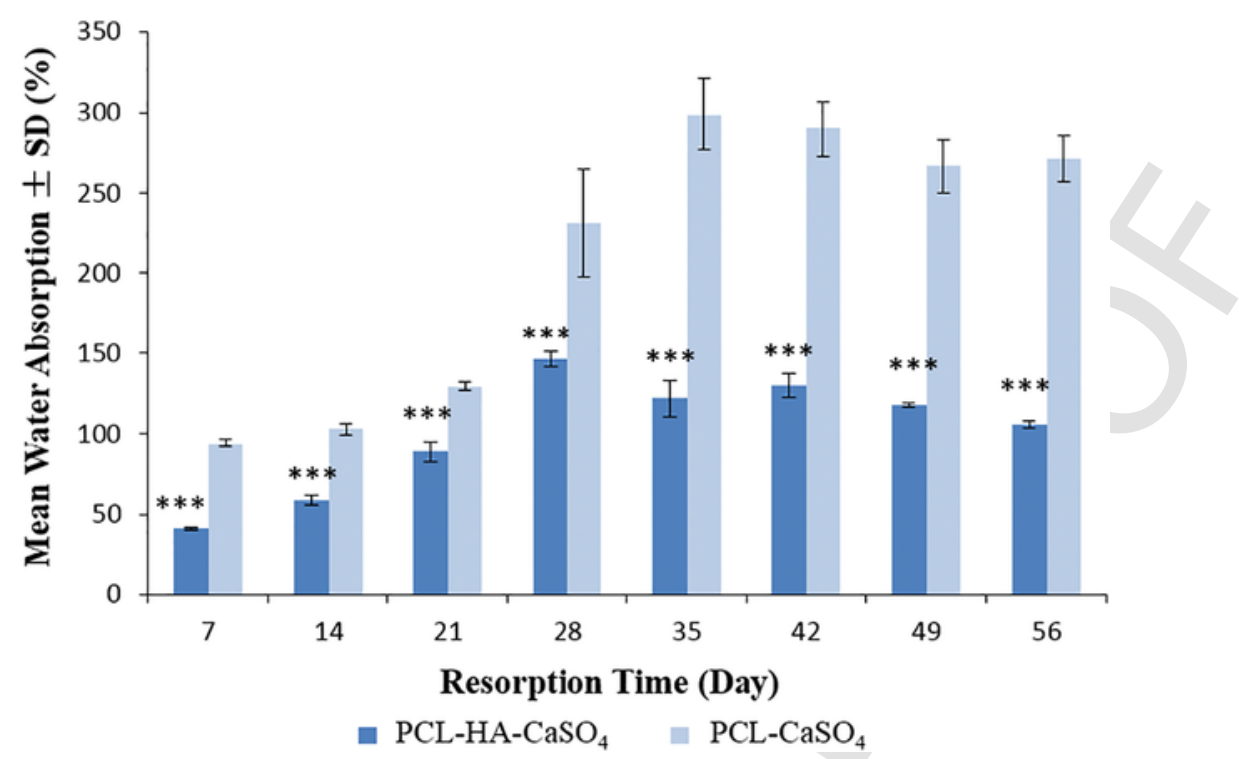

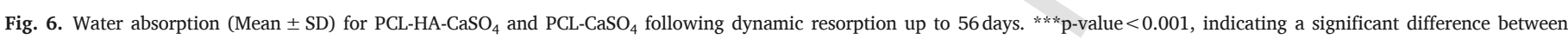
PCL-HA-CaSO ${ }_{4}$ and PCL-CaSO ${ }_{4}$ at the same time point.

$\begin{array}{lllllllllll}3 & 2 & 2 & 3 & 3 & 3 & 3 & 3 & \text { Day } 56\end{array}$

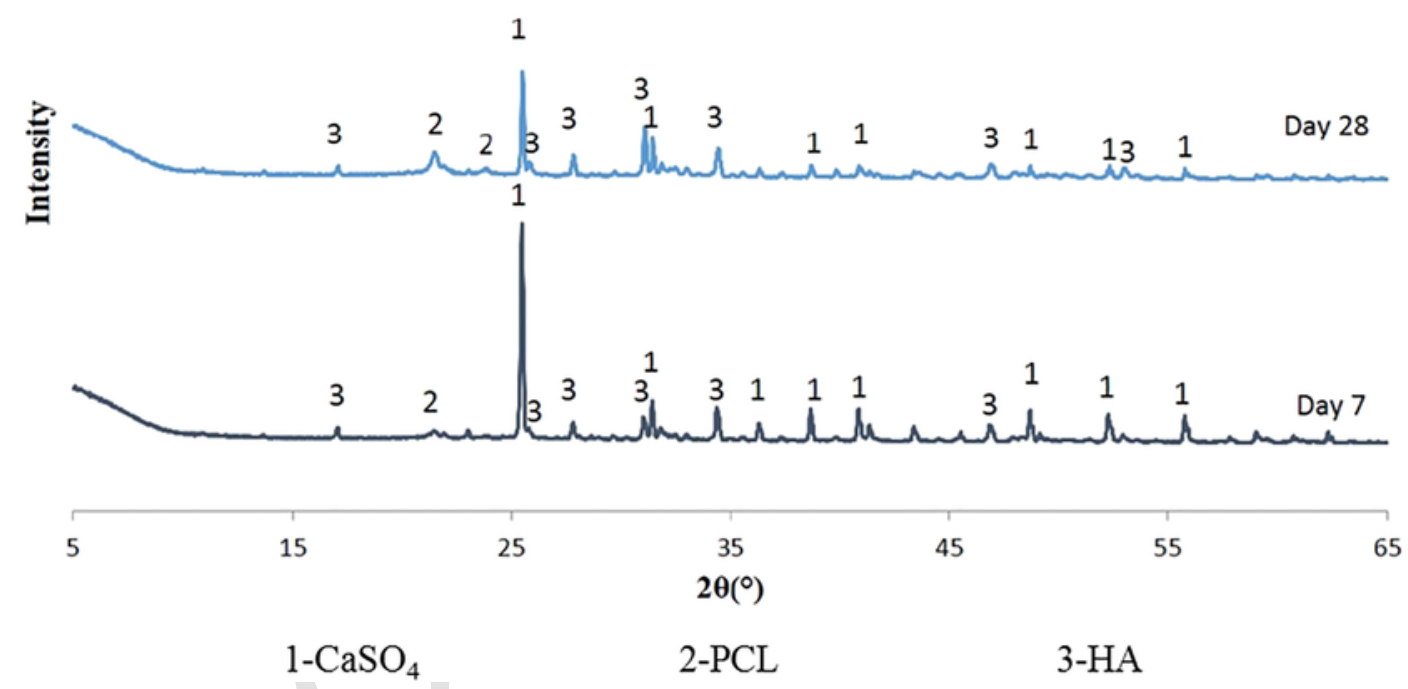

Fig. 7. XRD spectra for PCL-HA-CaSO ${ }_{4}$ after Day 7,28 , and 56 of dynamic in vitro resorption.

and placed in the fume cupboard under dry, low-pressure conditions for $48 \mathrm{~h}$ to facilitate solvent evaporation [26]. The untreated and PCL-treated scaffolds were classified as $\mathrm{HA}_{-} \mathrm{CaSO}_{4}$ and PCL-HA-CaSO respectively. Additionally, $\mathrm{CaSO}_{4}$ scaffolds were prepared using the same 3DP process and PCL treatment (PCL-CaSO ${ }_{4}$ ) to enable direct comparison during characterisation of the resorption behaviour.

\subsection{Compressive properties}

Compressive properties of HA-CaSO ${ }_{4}$ and PCL-HA-CaSO ${ }_{4}$ were determined using a universal materials test system (EZ50, Lloyds Instruments, UK). A $1 \mathrm{kN}$ load cell (XLC 01/2419, Lloyds Instruments, UK) was used, and the tests were performed at a rate of displacement of
$0.5 \mathrm{~mm} / \mathrm{min}$. The load cell had a load measurement accuracy of $\pm 0.5 \%$ and could read down to $1 / 200$ th of its capacity. A total of four scaffolds were tested for each material composition. Each scaffold was tested to failure, which was denoted when the load in the post-peak region had reduced to $80 \%$ of the peak load. One thousand force- $v s$.-displacement data points were then logged for each specimen. The compressive strength was defined as the maximum load recorded, divided by the apparent cross-section area of the scaffold. The compressive modulus was determined by measuring the maximum slope within the linear region of the stress- $v s$.-strain curve immediately after the toe-in region. Simpson's Rule was used to determine the compressive toughness, which was denoted as the area under the compressive stress- $v s$.-strain curve to the point of failure. 
a.

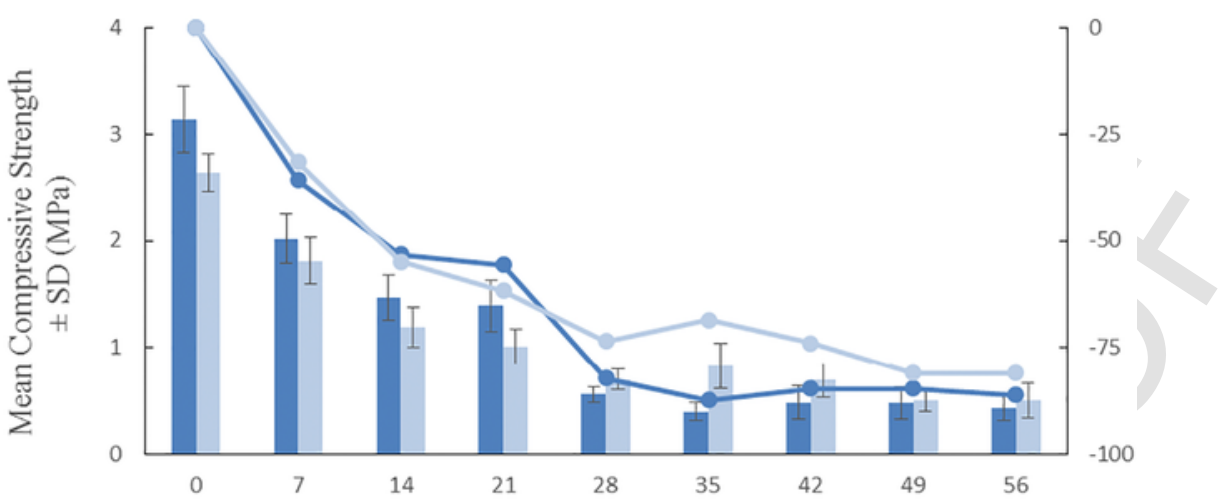

b.

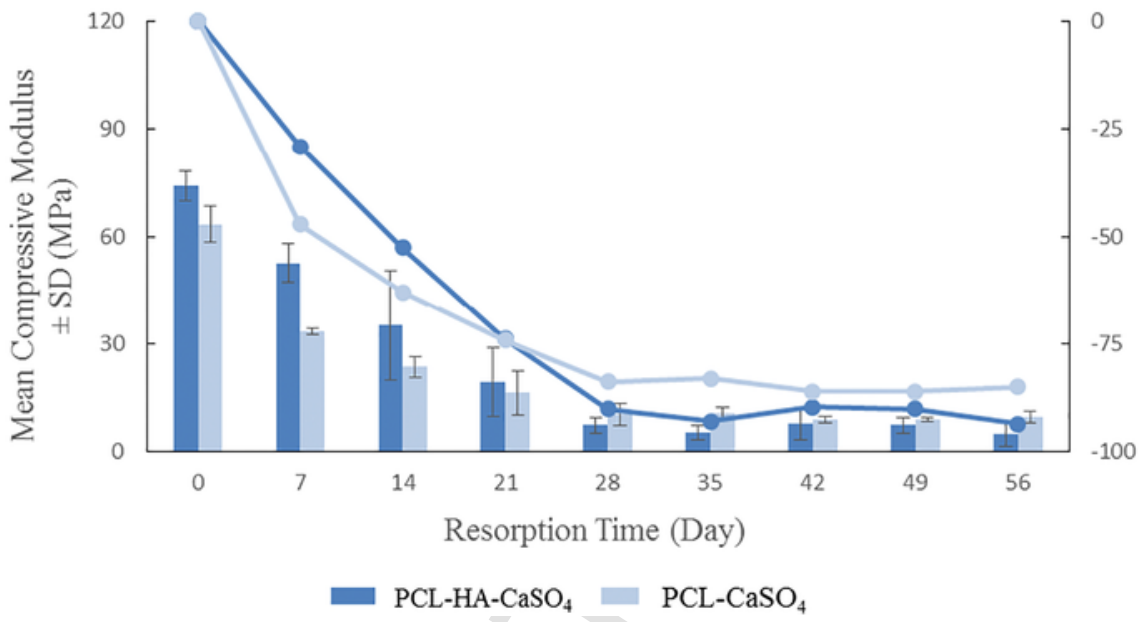

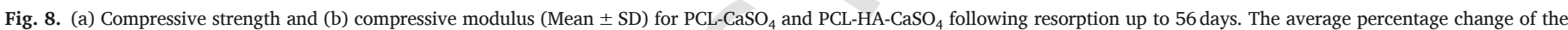
properties at each time point is also shown as a line plot referring to the labelled secondary Y-axis.

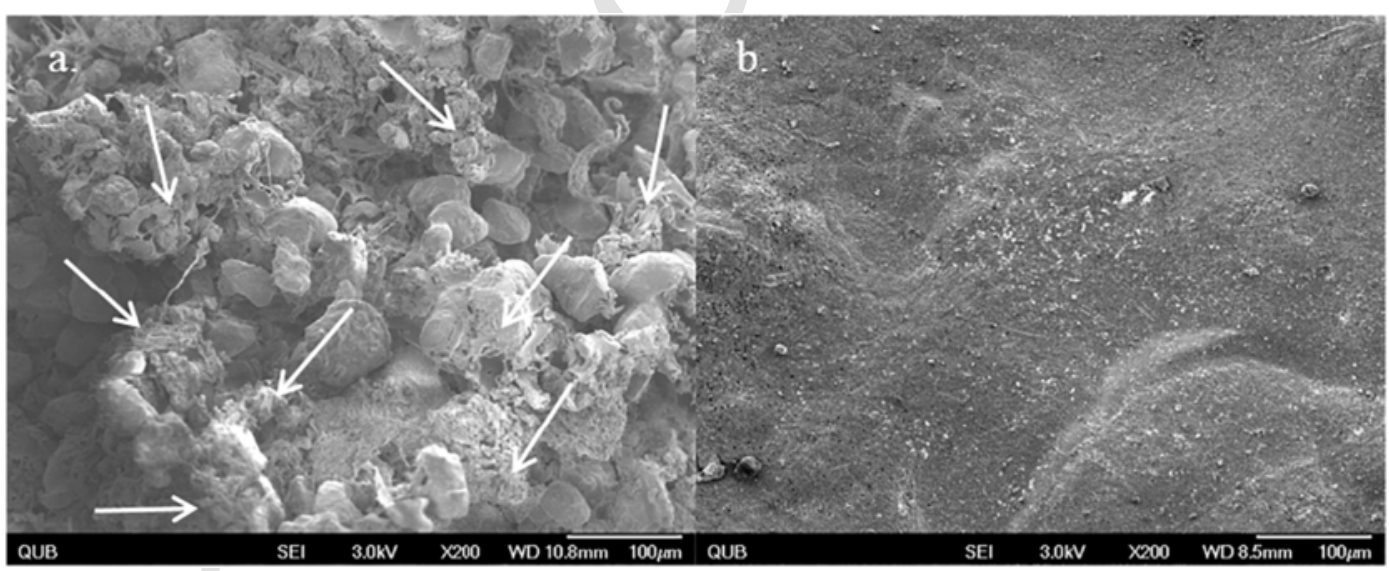

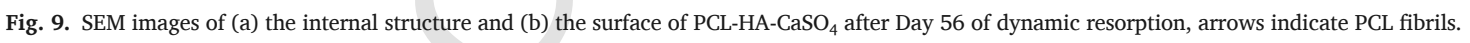

\subsection{Morphology}

3DP structures were sectioned longitudinally to facilitate observation of the internal architecture and morphology using field emission scanning electron microscope (SEM) (JEOL JSM-6500F, JEOL Ltd., Japan) at an operating voltage of $5 \mathrm{keV}$. Each structure was mounted on an aluminium stub using a cold cure resin (Extec Corp, Enfield, CT 06083-1258, US), allowed to cure for $24 \pm 2 \mathrm{~h}$ and subsequently gold-coated using a sputter chamber prior to SEM examination.

\subsection{In vitro resorption properties}

A high-throughput system was manufactured to facilitate the measurement of the in vitro resorption properties of 12 groups of $4 \mathrm{spec}$ imens simultaneously (Fig. 1). Within the system, the physiological medium was circulated using a 12-channel peristaltic pump in an effort to create dynamic flow conditions akin to the human body. Each channel controlled the flow rate of the medium which was connected to each reservoir and chamber. Each reservoir was sealed and placed in a temperature-controlled water bath to maintain the temperature of Tris- $\mathrm{HCl}$ buffer solution at $37 \pm 1{ }^{\circ} \mathrm{C}$. Each chamber was designed to con- 


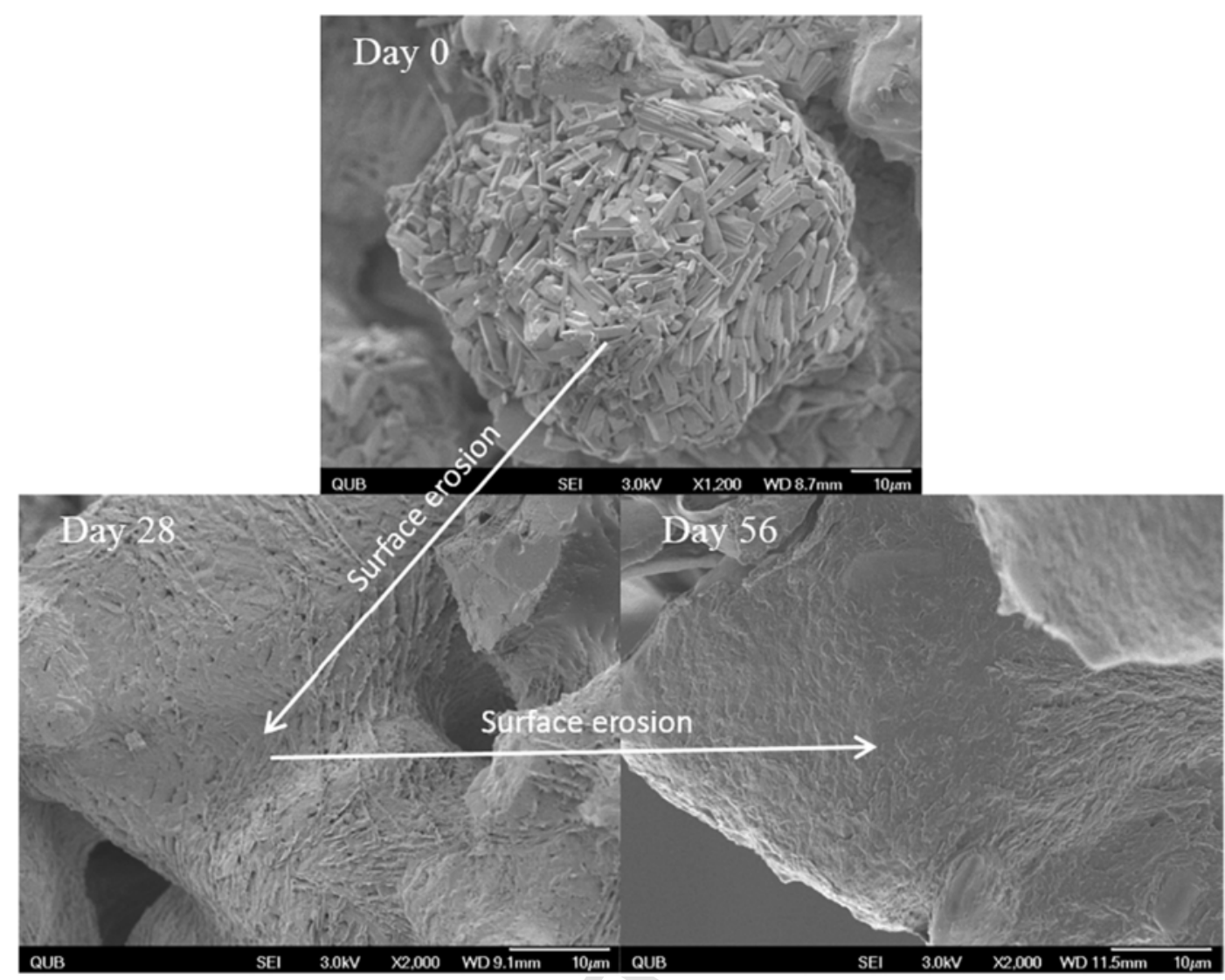

Fig. 10. SEM images of the ceramic surfaces on the internal structure of PCL-HA-CaSO ${ }_{4}$ after Day 0, 28, and 56 of dynamic resorption.

tain a group of scaffolds $(n=4)$. The buffer solution $(240 \mathrm{~mL})$, circulated throughout the system and refreshed weekly, was equally distributed to each 3DP scaffold within the group. It was comparable to the volume of medium (i.e. $60 \mathrm{~mL}$ ) used in previous static in vitro resorption studies [26].

The in vitro resorption behaviour of $\mathrm{HA}_{-} \mathrm{CaSO}_{4}, \mathrm{PCL}-\mathrm{HA}-\mathrm{CaSO}_{4}$, and PCL-CaSO ${ }_{4}$ under a dynamic flow condition was evaluated using the aforementioned system. The rotational speed of the peristaltic pump was $4.25 \mathrm{RP}$, which equated to a circulating flow rate of approx. $1 \mathrm{~mL} /$ min [27]. This flow rate has previously been used for the culturing of engineered bone in perfusion bioreactors $[27,28]$. Note that the actual flux that 3DP scaffolds experienced may be slightly different from the setup flow rate as it also depended on other factors, such as pore size. The resorption properties were measured over a 56-day period. Every week, a group of scaffolds were removed and characterised.

The wet mass was measured after carefully removing all the excess water from the structure using sterile filter paper. Each scaffold was then rinsed with deionised water and dried in an oven at $37 \pm 1{ }^{\circ} \mathrm{C}$ for $48 \mathrm{~h}$. The dry mass was then measured. The water absorption (wt $\%$ ) and mass change (\%) before and after immersion in buffer solution were calculated using Eqs. (1) and (2):

$$
\begin{aligned}
& \text { Water absorption }(\%)=\frac{\mathrm{m}_{\mathrm{t}, \mathrm{w}}-\mathrm{m}_{\mathrm{t}, \mathrm{d}}}{\mathrm{m}_{\mathrm{t}, \mathrm{d}}} \times 100 \\
& \text { Mass change }(\%)=\frac{\mathrm{m}_{0, \mathrm{~d}}-\mathrm{m}_{\mathrm{t}, \mathrm{d}}}{\mathrm{m}_{0, \mathrm{~d}}} \times 100
\end{aligned}
$$

where: $\mathrm{m}_{0, \mathrm{~d}}=$ dry mass before immersion in buffer solution $(\mathrm{g})$; $\mathrm{m}_{\mathrm{t}, \mathrm{d}}=$ dry mass after immersion in buffer solution $(\mathrm{g})$;

$\mathrm{m}_{\mathrm{t}, \mathrm{w}}=$ wet mass after immersion in buffer solution $(\mathrm{g})$.
The compressive properties were also determined for each scaffold following drying as previously described. Although determining the compressive properties of the 3DP structures under wet conditions would have been beneficial as this is more representative of the in vivo environment. Notwithstanding this fact, drying each of the 3DP structures prior to mechanical testing was chosen as to allow for the non-destructive characterisation to be undertaken beforehand.

$\mathrm{X}$-ray diffraction (XRD) analysis was conducted on the different scaffold types using an X-Pert Pro X-ray diffraction system with an X'Celerator X-ray detector (PANalytical Ltd., UK). X'Pert High Score software was used to identify the phases present in the different scaffold types at Day $0,7,28$, and 56 .

Each scaffold type was imaged using SEM to observe the effects of resorption on the microstructure and morphology at Day $0,7,28$, and 56. X-ray microtomography ( $\mu$-CT) was also used to determine the structural evolution of the 3DP structures in the dry condition as a function of in vitro resorption using a SkyScan 1174 compact desktop X-ray micro-tomography ( $\mu$-CT) scanner system (SkyScan N.V., Belgium). Specifically, scanning was conducted at medium resolution (600 axial $\mu$-CT slices with $1024 \times 1024$ pixels bitmap image, $16.25 \mu \mathrm{m}$ pixel size). The micro-focus X-ray source operated at a voltage of $50 \mathrm{kV}$ and a current of $800 \mu \mathrm{A}$. Aluminium filters $(0.75 \mathrm{~mm})$ were applied for beam hardening reduction. During the scanning process the specimen stage was rotated over $360^{\circ}$ at a rotation step of 0.51 . At each rotation step an angular shadow projection of the specimen was acquired at an exposure time of $5.5 \mathrm{~s}$. The X- ray shadow projections were then digitised and the acquisition geometry for each scan was extracted from the dataset of transmission images using a reconstruction programme (i.e. smoothing $=4$, ring artefact correction $=14$ and beam hardening correction $=46 \%$ ). Grayscales values are proportional to the material density [29]. One of the challenges of analysing changes in the struc- 


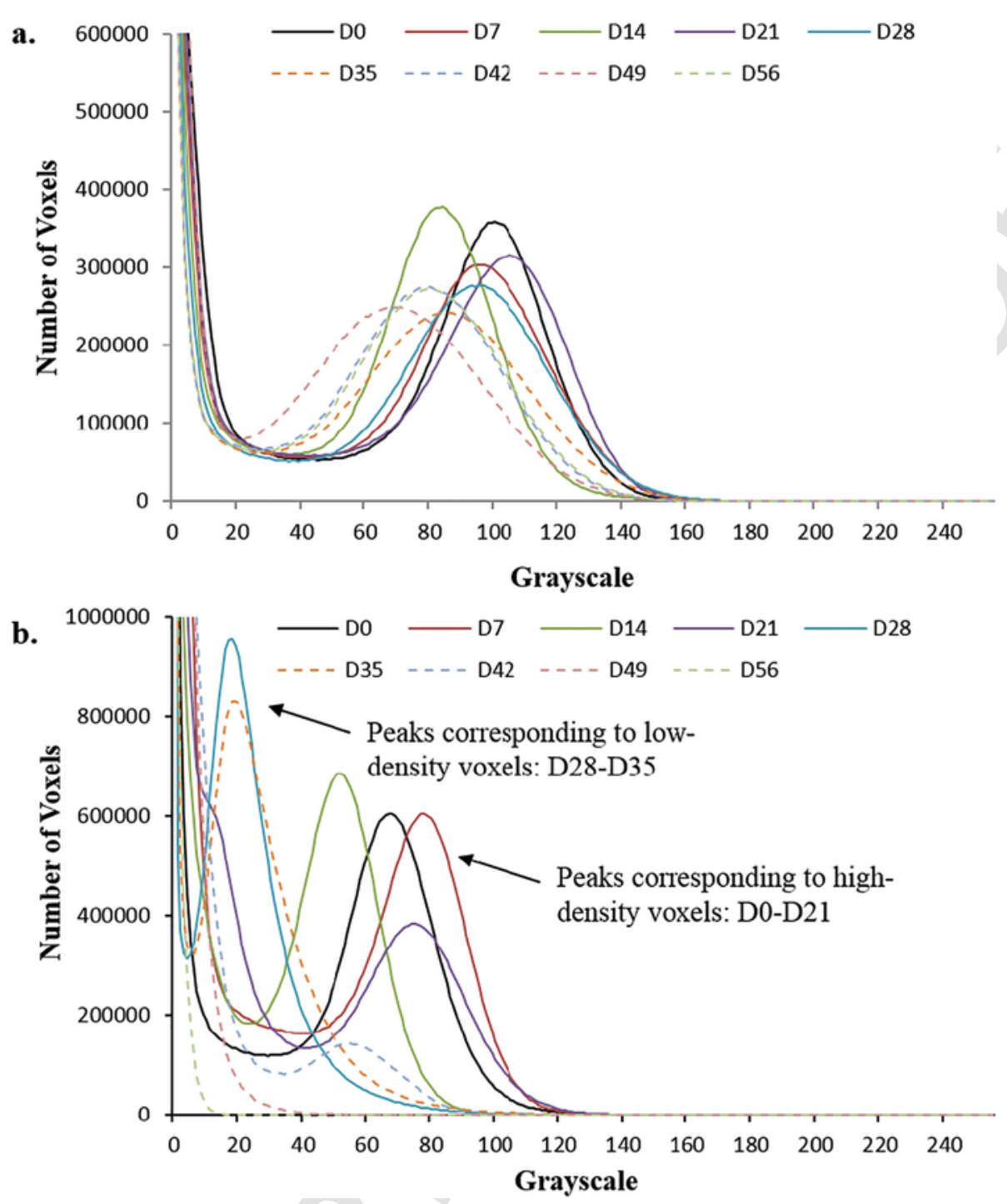

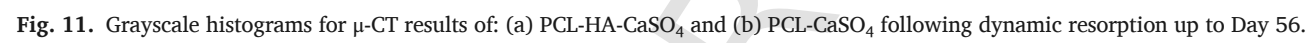

tural properties as a function of resorption time was the continuous shift in the X-ray absorption peaks on the grayscale histogram due to density reduction. Therefore, it was difficult to use the same threshold level for materials demonstrating different resorption behaviour. The upper and lower threshold levels for reconstructed cross-sectional images were determined from the grayscale histogram, which was generated using ImageJ software (National Institutes of Health, USA). The threshold levels were selected in positions that best separated materials from empty spaces as well as one material phase from another if distinct peaks were indicated from the histogram. Thereafter, the structural properties of interest (e.g. volume and degree of porosity) were determined using the SkyScan CT-analyser software (Version1.10.1.0, SkyScanN.V., Belgium). Further morphological analysis on the reconstructed image dataset was performed using ImageJ software (National Institutes of Health, USA) with the BoneJ plugin [30].

\subsection{Statistical analysis}

Data collected from each experimental test was evaluated for statistical significance using SPSS13.0 software (SPSS, USA). Differences between treatment groups were assessed using one-way Analysis of Variance (ANOVA) with post-hoc Bonferroni correction. A p-value $<0.05$ denoted statistical significance. Data analysis was selected on basis of normal probability tests.

\section{Results and discussion}

\subsection{Compressive properties}

PCL-HA-CaSO ${ }_{4}$ exhibited significantly higher compressive properties $(\mathrm{p}<0.05)$ than $\mathrm{CaP}_{-} \mathrm{CaSO}_{4}$ (Fig. 2). One of the major drawbacks associated with scaffold manufacture using powder-based 3D printing technology is poor mechanical performance due to high inter-particle spaces within the printed structures [31]. The resulting stress concentrations yield rapid and catastrophic failure under compressive forces (Fig. 3a). Compressive loading is the most common mode of loading applied to bone during normal day-to-day activities. Therefore, it is essential to improve the compressive properties of tissue engineered bone scaffolds. The most rational approach to overcome this inherent drawback associated with 3DP scaffolds was to fill the inter-particle spaces using a biocompatible and bioresorbable material following 3D printing. Previous studies have used biopolymers as infiltration materials to improve the mechanical properties of 3DP bioceramic scaffolds [26,32-33]. PCL infiltrating the micropores of the ceramic scaffold increased the interfacial contact area providing a corresponding increase in the 


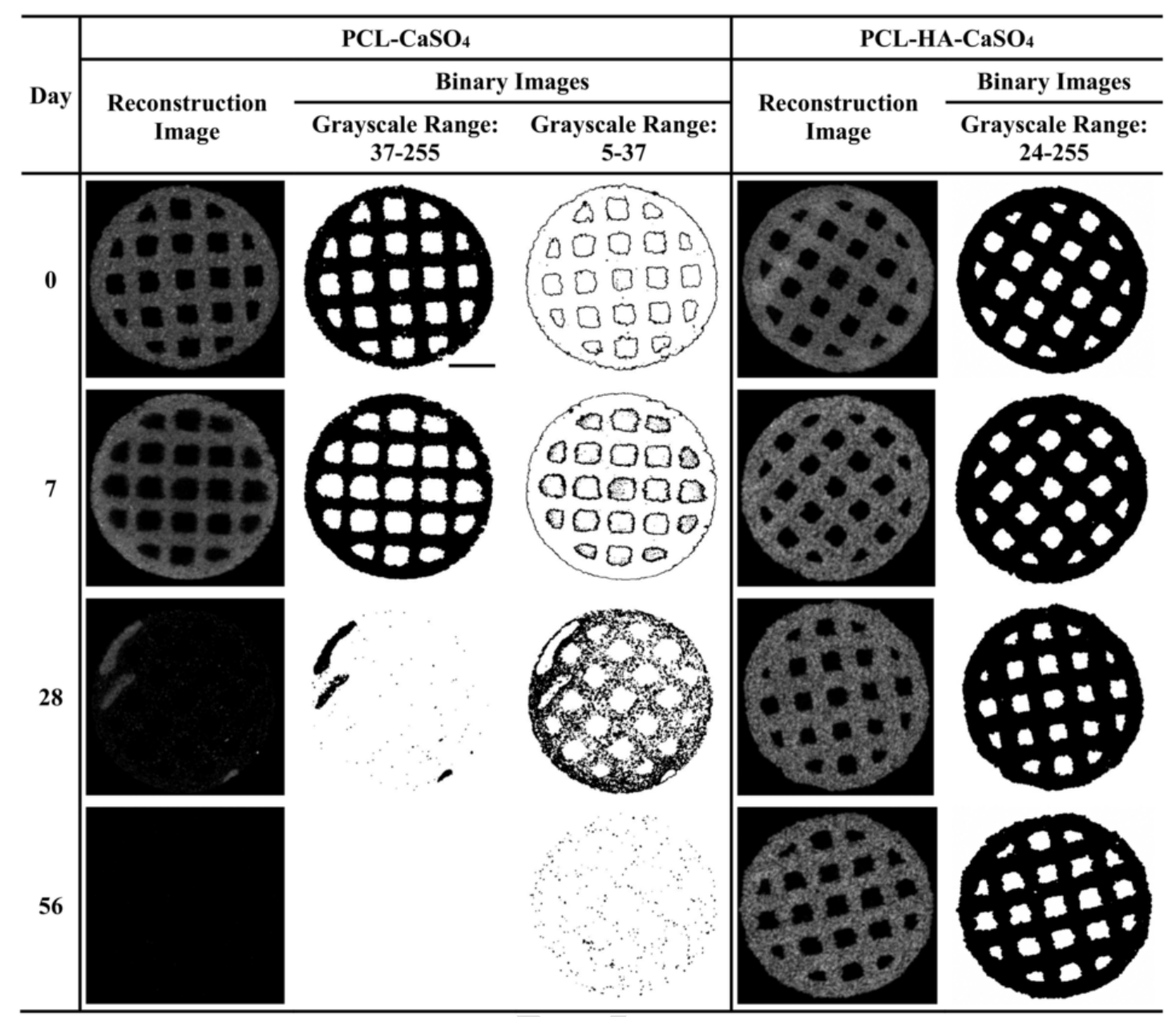

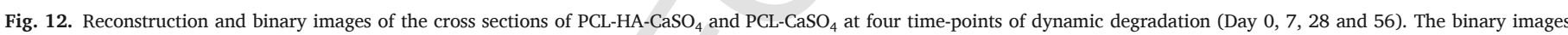

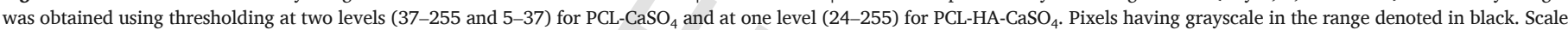
bar $=2 \mathrm{~mm}$.

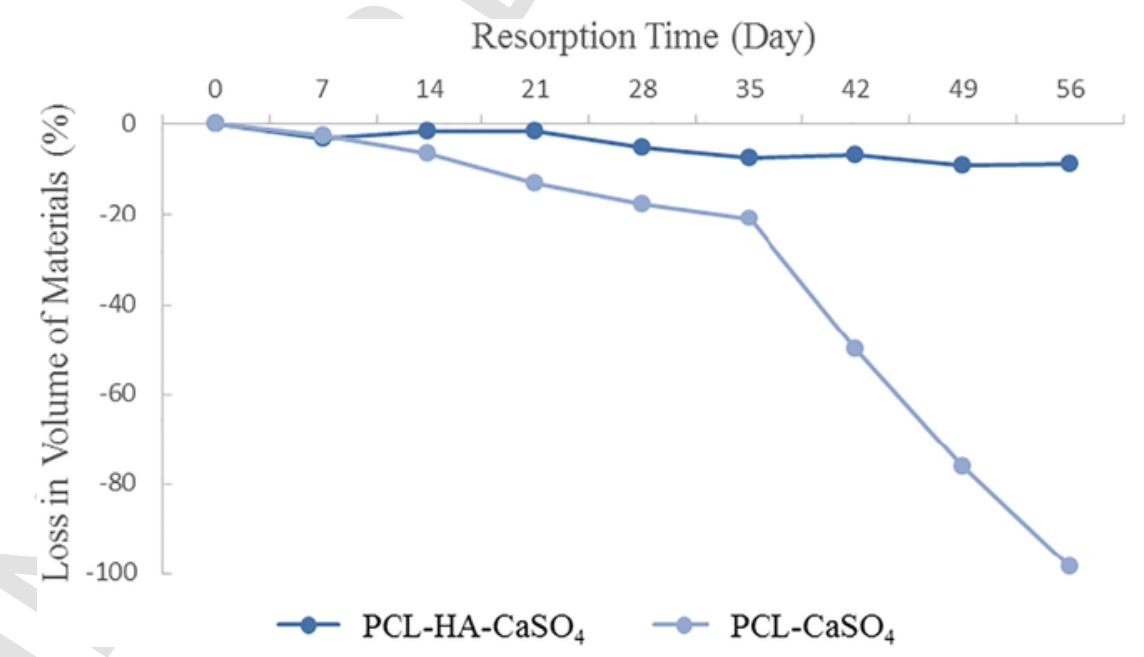

Fig. 13. Results determined by $\mu$-CT analysis for material volume loss of PCL-HA-CaSO ${ }_{4}$ and PCL-CaSO ${ }_{4}$ scaffolds as a function of dynamic resorption up to Day 56 .

compressive properties of the scaffold. In this study, the compressive strength, compressive modulus and toughness of $\mathrm{HA}-\mathrm{CaSO}_{4}$ was significantly increased $(\mathrm{p}<0.001)$ from $0.84 \pm 0.06 \mathrm{MPa}, 13.64 \pm 1.76 \mathrm{MPa}$, and $0.03 \pm 0.00410^{-6} \mathrm{~J} / \mathrm{m}^{3}$ to $2.64 \pm 0.18 \mathrm{MPa}$ (average $314 \% \uparrow$ ), $63.39 \pm 4.92 \mathrm{MPa}$ (average $465 \% \uparrow$ ), and $0.26 \pm 0.0910^{-6} \mathrm{~J} / \mathrm{m}^{3}$ (average $\quad 867 \% \quad \uparrow$ ) following PCL infiltration.
The infiltrated PCL-HA-CaSO ${ }_{4}$ scaffold demonstrated high structural integrity following compressive loading (Fig. 3b). SEM analysis showed the PCL covered the surface pores and also infiltrated deep into the inter-particle spaces (Fig. 4). Consequently, the PCL increased the overall density of 3DP scaffolds, connected the adjacent powder particles, and bridged cracks in the form of fibrils during compressive loading. 


\subsection{In vitro resorption properties}

The purpose of an in vitro resorption experiment was to gain a better understanding of material resorption trends using methods analogous to in vivo conditions. Resorption under static conditions is the typical methodology used to evaluate the resorption properties because it is easier to implement. However, implants are not exposed to static conditions in vivo, but rather a thermodynamically open environment that involves the dynamic flow of body fluid [34]. Therefore, it is more representative to subject 3DP scaffolds to in vitro resorption under dynamic flow conditions.

In this study, $\mathrm{HA}-\mathrm{CaSO}_{4}$ scaffolds were too fragile to handle following 7 days immersion in the buffer solution. The $\mathrm{HA}-\mathrm{CaSO}_{4}$ scaffold type demonstrated a high specific surface area due to high inter-particle spaces. When subjected to an abrasive attack from the dynamic flow, this resulted in a rapid deterioration of structural properties. In contrast, the PCL-HA-CaSO ${ }_{4}$ scaffold type maintained increased structural integrity following 56 days of immersion in the buffer solution. Therefore, it was pertinent for the remainder of the study to focus on the behaviour of the PCL-HA-CaSO ${ }_{4}$ 3DP scaffold type as oppose to its $\mathrm{PCL}_{-} \mathrm{CaSO}_{4}$ counterpart.

PCL-HA-CaSO ${ }_{4}$ demonstrated a relatively fast mass loss from Day 0-28 (-48.65 $\pm 0.80 \%$ ) (Fig. 5). Following Day 28, further mass loss continued at a slower rate $(-54.48 \pm 0.54 \%)$ towards Day 56 . PCL-HA-CaSO $_{4}$ exhibited significantly lower mass loss than PCL-CaSO between Day 28 and Day 56 ( $\mathrm{p}<0.05$ ). The use of continuous dynamic flow removed ceramic dissolution by-products from the 3DP scaffold and reduced the potential for local ion oversaturation. Oversaturation of ions has the potential to confound the resorption process. During the first 28 days of in vitro resorption, a strong linear correlation $\left(\mathrm{R}^{2}=0.996\right)$ was observed between mass loss and resorption time, suggesting a constant dissolution rate. The rapid resorption of PCL-HA-CaSO ${ }_{4}$ following 28 days of immersion in buffer solution was related to the $\mathrm{HA}$ and $\mathrm{CaSO}_{4}$. It was speculated that ceramics were readily dissolved until a time when the remaining ceramic particles were encapsulated with PCL. Consequently, the rate of further resorption receded.

Water absorption of PCL-HA-CaSO 4 also increased rapidly from Day 0-28 (146.36 $\pm 4.93 \%$ ) (Fig. 6). Following Day 28, a gradual reduction in water absorption was observed. After 56 days of immersion in buffer solution, the PCL-HA-CaSO ${ }_{4}$ scaffold exhibited water absorption of $106.11 \pm 1.92 \%$. It was significantly lower $(\mathrm{p}<0.05)$ than the $\mathrm{PCL}^{-\mathrm{CaSO}_{4}}$ 3DP scaffold throughout the testing period. Water absorption was a balance between removal of hydrophilic resorbing material and the water uptake of surface flaws, which were created due to material removal. The local regions where ceramic particles were exposed to buffer solution became vulnerable due to dissolution. The dynamic flow demonstrated sufficient shear forces to dislodge ceramic particles from weakened regions, which created cavities on the surface of the 3DP scaffold. These cavities are likely to have trapped buffer solution and increased water absorption between Day 0 and Day 28, which represented the period when the fastest mass loss was evident. Consequently, the proportion of PCL increased (Fig. 7). It is a more hydrophobic material when compared to $\mathrm{HA}$ or $\mathrm{CaSO}_{4}$, and therefore the rate of water absorption of PCL-HA-CaSO 4 slowed after Day 28.

The compressive strength of PCL-HA-CaSO ${ }_{4}$ decreased by $73.48 \%$ from between Day $0(2.64 \pm 0.18 \mathrm{MPa})$ and Day $28(0.70 \pm 0.10 \mathrm{MPa})$ (Fig. 8). Thereafter, there was no significant change in the compressive strength until Day $56(0.50 \pm 0.16 \mathrm{MPa})$. After 7 days of immersion in buffer solution, the compressive modulus rapidly reduced to $37 \%$ of the original compressive modulus. However, there was no significant change $(\mathrm{p}>0.05)$ in the compressive modulus following Day 14 . On average, the 3DP scaffold retained $15 \%$ of its original compressive modulus after 56 days of immersion in the buffer solution. The com- pressive properties for PCL-CaSO ${ }_{4}$ subjected to resorption followed a similar trend. However, the reduction in both compressive strength and compressive modulus for $\mathrm{PCL}^{-\mathrm{CaSO}_{4}}$ was greater than those for PCL-HA-CaSO 4 after Day 21. The compressive properties were affected by the material removal from the 3DP scaffold structure. The greater resorption between Day 0 and Day 28 resulted in a significant reduction in compressive properties ( $\mathrm{p}<0.05)$.

PCL was selected as the infiltration material because it can provide long-term mechanical stability to withstand the forces exhibited during the early stages of wound contraction and bone healing owing to its relatively slow resorption profile in contrast to other biopolymers, e.g. poly(lactic-co-glycolic acid) (PLGA) [35]. Additionally, PCL offers better ductile characteristics when compared to poly-L-lactic acid (PLLA), which can help augment the brittle nature of the $\mathrm{HA}-\mathrm{CaSO}_{4}$ and therefore improve its fracture resistance and structural retention during physical loading $[26,36]$. An important issue to consider when using a solvent (e.g. chloroform) to dissolve the PCL is to ensure its complete removal before clinical application as this will be critical to the cellular behaviour of the PCL coated 3DP scaffolds. Peroligo et al. (2007) used differential scanning analysis to confirm that full evaporation of the chloroform from a PCL coated bioceramic scaffold structure can be achieved within 24-48 h [37]. They concluded that full evaporation of the chloroform was achieved within this time frame since a relatively thin coating of PCL with a high surface area was used - which was also the case in this investigation. Additionally, Tarafder and Bose (2014) used a solvent to dissolve PCL prior to coating tricalcium phosphate 3DP scaffolds and reported no adverse effect of PCL on the biocompatibility of the scaffold or its in vivo bone forming capabilities after six weeks implantation [38].

The diffraction peaks of the $\mathrm{CaSO}_{4}$ from the XRD spectra for the PCL-HA-CaSO 4 attenuated greatly during in vitro resorption (Fig. 7). The $\mathrm{CaSO}_{4}$ phase (1) was difficult to detect after Day 56 and HA (3) became the dominant inorganic phase detected. The organic phase, PCL (2), also remained within the 3DP scaffold structure, exhibiting diffraction peaks at $2 \theta=21.3^{\circ}$ and $23.7^{\circ}$. This result indicated that $\mathrm{CaSO}_{4}$ experienced a more intensive level of dissolution when compared to the HA phase. It is well known that $\mathrm{CaSO}_{4}$ dissolves more rapidly than $\beta$-TCP and HA [39]. The loss of scaffold mass was attributed to the steady dissolution of $\mathrm{CaSO}_{4}$, which was exposed directly to the buffered solution. The remaining $\mathrm{CaSO}_{4}$ was encapsulated by PCL and therefore, was unable to be detected towards the end of resorption. The absence of $\mathrm{CaSO}_{4}$ during the latter stages of resorption could provide several benefits: firstly, its rapid dissolution released a large amount of $\mathrm{Ca}^{2+}$ ions, which would favour cell proliferation in vivo [40-41]. Secondly, the removal of the $\mathrm{CaSO}_{4}$ left behind empty spaces that could enhance new bone formation [42]. HA remained almost passive because the resorption of multiphase-ceramic always begins with the dissolution of the most soluble phase [42]. The greater dissolution of $\mathrm{CaSO}_{4}$ created an environment rich in $\mathrm{Ca}^{2+}$ ions, which also inhibited HA dissolution. The SEM analysis showed that a large number of ceramic particles remained within the 3DP scaffold at Day 56, which were connected by PCL fibrils (Fig. 9). Additionally, the scaffold surface was still well covered with the PCL coating layer. Therefore, a high level of structural integrity was still available after the 3DP scaffold was subjected to dynamic in vitro resorption for 56 days. This signifies the potential for the 3DP scaffold to offer structural and mechanical support to the newly formed tissues.

During the 3D printing process, a solid structure was formed due to the precipitation of ceramic aggregates when the setting reaction between the $\mathrm{CaSO}_{4}$ powder and water-based binder occurred (Fig. 10). The aggregated rod-like crystals eroded when the structure was subjected to resorption in the buffer solution. The surface erosion resulted in a smoother topography, which was observed for the PCL-HA-CaSO morphology after 28 days immersion in buffer solution. More signifi- 
cant erosion was demonstrated when the resorption proceeded to Day 56. These results demonstrated that the mechanism of surface erosion was dominant, whereby the material located within the internal structure only resorbed following complete resorption of the external surfaces.

One of the challenges of analysing resorption using $\mu$-CT is the continuous shift of the X-ray absorption peaks on the grayscale histogram, which was proportional to the material density [29]. A decrease in material density related to the microporosity created as a consequence of the resorption process. A voxel containing an element of microporosity exhibited a relatively low grayscale value. A more dramatic shift of the X-ray absorption peaks towards lower values was observed on the grayscale histogram of PCL-CaSO ${ }_{4} 3 \mathrm{DP}$ scaffolds compared to their PCL-HA-CaSO ${ }_{4}$ counterparts (Fig. 11). There was no distinct peak observed on the histogram for PCL-CaSO ${ }_{4}$ 3DP scaffolds between Day 42 and Day 56. To demonstrate material distribution at different stages of the resorption period, binary images of cross-sections of 3DP scaffolds were generated via thresholding the reconstruction images at different grayscale levels based on the histograms: (1) 24-255 for PCL-HA-CaSO and (2) 5-37 and 37-255 for PCL-CaSO 4 (Fig. 12). The cross-sectional images of PCL-HA-CaSO 4 after 56 days of resorption did not exhibit a significant change and a high level of structural integrity was maintained. Smaller pores (approx. $100 \mu \mathrm{m}$ ) were created within the structure after 56 days of resorption. However, $\mathrm{PCL}-\mathrm{CaSO}_{4}$ scaffolds experienced a rapid loss of material (Fig. 13), which correlated to a lower grayscale range at Day 28 as a consequence of the reduction in density throughout the 3DP scaffold. This transformation continued to be more significant as only a few materials were observed on the binary images at Day 56. The dip-coating technique enabled deep infiltration of PCL within the scaffold structure, which efficiently filled the inter-particle spacing. The entire 3DP scaffold structure was maintained due to the PCL demonstrating a low level of resorption by Day 56 . Due to this infiltration of PCL within the 3DP scaffold it was highly unlikely that large spaces were created unless the in vitro resorption reached a relatively high level, which was the case for the PCL-CaSO ${ }_{4} 3 \mathrm{DP}$ scaffold type. However, for the PCL-HA-CaSO ${ }_{4}$ based 3DP scaffold, both PCL infiltration and faster dissolution of $\mathrm{CaSO}_{4}$ reduced HA dissolution. Consequently, a large amount of HA particles remained within the 3DP scaffold structure.

This study has been focused on the printability of the polymer coated composite materials and the corresponding in vitro data reported over a 56-day period shows promise. Notwithstanding this fact, there may be additional questions that are still unresolved and areas for further development. Although, use of the PCL coating successfully increased the compressive strength to match cancellous bone (2-12 MPa) [3], the compressive modulus was still lower than that of cancellous bone (100-500 MPa) due to the loose packing nature of ceramic particles. Further improvement in the compressive properties could be achieved through: (1) optimisation of the particle characteristics to achieve more dense powder packing, or (2) incorporation of ceramic particles within the polymer infiltration solution for better structural reinforcement. Further investigation is necessary to understand how the PCL-HA-CaSO4 based 3DP scaffolds perform under cyclic loading within a pseudo-physiological environment as this is an essential next step before in vivo testing using an appropriate animal model. Studies regarding the cytotoxic response of both $\mathrm{HA}$ and $\mathrm{CaSO}_{4}$ based biomaterials have documented favourable findings corroborating their potential in orthopaedic applications $[14,25,38,44-46]$. However, there may be toxicity issues associated with our composite scaffold since the materials were processed via a novel technology. It is important that compressive and tuneable resorption properties of the 3DP scaffolds are appropriately balanced to the sequence of events exhibited during new bone formation in vivo, i.e. encouraging new tissue growth during the initial stages of implantation, offering sufficient structural and mechanical support, and following an appropriate resorption profile during the bone healing phase. This balance can be achieved by varying the $\mathrm{CaSO}_{4}$ :CaP:PCL of the final material formulation to mimic the mechanical properties and resorption requirements for a specific clinical application, which will vary considerably depending on a number of factors, e.g. the age of patient, bone quality, size, and anatomical site of the defect. Further work is currently ongoing to (1) augment the mechanical properties of the existing 3DP scaffolds and understand how they perform under cyclic loading within a pseudo-physiological environment, (2) determine the extent of biocompatibility and osteogenic properties of these 3DP manufactured scaffolds using an appropriate in vivo animal model, and (3) understand if the 3DP scaffolds offer the necessary mechanical and structural support and resorption rate required for complete repair of a critical sized bone defect and coincide with the sequence of events involved in natural bone healing.

\section{Conclusion}

This study demonstrated that a blend of $\mathrm{HA}$ and $\mathrm{CaSO}_{4}$ was a reliable formulation for powder-based 3DP technology. The combination of using a fast resorbing $\mathrm{CaSO}_{4}$ powder and the relatively slower HA powder represents a promising prospect for tuning the bioresorption of 3DP scaffolds. The HA-CaSO ${ }_{4}$ could be tailored to coincide with tissue growth rate for different surgical procedures. Following manufacture of the 3DP scaffold, PCL infiltration was used to successfully fill the inter-particle spacing within the scaffold and as a result, the compressive properties increased significantly. The in vitro resorption properties under a dynamic flow conditions demonstrated PCL inhibited HA dissolution and maintained the overall structural integrity after 56days of resorption. The remaining scaffold structure accounted for $45.52 \pm 0.54 \%$ of the original mass. Rapid reduction in the compressive properties during the first 28 days were mainly attributed to $\mathrm{CaSO}_{4}$ dissolution. The majority of the $\mathrm{CaSO}_{4}$ powder resorbed within 56 days, while HA remained almost passive. The resorption of the $\mathrm{CaSO}_{4}$ particles allowed for spaces to become available, which could act as niches, encouraging new tissue growth in the vicinity of the slow resorbing $\mathrm{HA}$. The prepared $\mathrm{HA}_{-} \mathrm{CaSO}_{4}$ scaffold with enhanced mechanical properties and tuneable bioresorption has potential as a good candidate for bone tissue engineering applications.

\section{References}

[1] B. Leukers, H. Gülkan, S.H. Irsen, S. Milz, C. Tille, M. Schieker, H. Seitz, Hydroxyapatite scaffolds for bone tissue engineering made by 3D printing, J. Mater. Sci. Mater. Med. 16 (12) (2005) 1121-1124.

[2] S.H. Irsen, B. Leukers, C. Höckling, C. Tille, H. Seitz, Bioceramic granulates for use in 3D printing: process engineering aspects, Mater. Werkst. 37 (6) (2006) 533-537.

[3] F. Peters, T. Hanel, A. Bernhardt, A. Lode, M. Gelinsky, H. Duerr, In vitro examination of 3D printed vs. milled scaffolds from beta-tricalcium phosphate (beta-TCP) for patient individual bone regeneration, In: Poster Session at the 8th World Biomaterials Congress, Amsterdam, Netherlands 2008, 2008.

[4] E. Vorndran, M. Klarner, U. Klammert, L.M. Grover, S. Patel, J.E. Barralet, U. Gbureck, 3D powder printing of $\beta$-tricalcium phosphate ceramics using different strategies, Adv. Eng. Mater. 10 (12) (2008) B67-B71.

[5] C. Bergmann, M. Lindner, W. Zhang, K. Koczur, A. Kirsten, R. Telle, H. Fischer, 3D printing of bone substitute implants using calcium phosphate and bioactive glasses, J. Eur. Ceram. Soc. 30 (12) (2010) 2563-2567.

[6] S. Rahmati, F. Shirazi, H. Baghayeri, Perusing piezoelectric head performance in a new 3-D printing design, Tsinghua Sci. Technol. 14 (2009) 24-28.

[7] Z. Zhou, F. Buchanan, C. Mitchell, N. Dunne, Printability of calcium phosphate: calcium sulfate powders for the application of tissue engineered bone scaffolds using the 3D printing technique, Mater. Sci. Eng. C 38 (2014) 1-10.

[8] J. Will, R. Melcher, C. Treul, N. Travitzky, U. Kneser, E. Polykandriotis, R. Horch, P. Greil, Porous ceramic bone scaffolds for vascularized bone tissue regeneration, J. Mater. Sci. Mater. Med. 19 (8) (2008) 2781-2790.

[9] R. Chumnanklang, T. Panyathanmaporn, K. Sitthiseripratip, J. Suwanprateeb, 3D printing of hydroxyapatite: effect of binder concentration in pre-coated particle on part strength, Mater. Sci. Eng. C 27 (4) (2007) 914-921.

[10] S.C. Cox, J.A. Thornby, G.J. Gibbons, M.A. Williams, K.K. Mallick, 3D printing of porous hydroxyapatite scaffolds intended for use in bone tissue engineering applications, Mater. Sci. Eng. C 47 (2015) 237-247. 
[11] Z. Zhou, F. Buchanan, A. Lennon, N. Dunne, Investigating approaches for three-dimensional printing of hydroxyapatite scaffolds for bone regeneration, Key Eng. Mater. 631 (2015) 306-311.

[12] H. Seitz, W. Rieder, S. Irsen, B. Leukers, C. Tille, Three-dimensional printing of porous ceramic scaffolds for bone tissue engineering, J. Biomed. Mater. Res. B. 74( (2) (2005) 782-788.

[13] S. Schaefer, R. Detsch, F. Uhl, U. Deisinger, G. Ziegler, How degradation of calcium phosphate bone substitute materials is influenced by phase composition and porosity, Adv. Eng. Mater. 13 (4) (2011) 342-350.

[14] L.F. Peltier, E.Y. Bickel, R. Lillo, M.S. Thein, The use of plaster of Paris to fill defects in bone, Ann. Surg. 146 (1) (1957) 61.

[15] G. Orsini, J. Ricci, A. Scarano, G. Pecora, G. Petrone, G. Iezzi, A. Piattelli, Bone-defect healing with calcium-sulfate particles and cement: an experimental study in rabbit, J. Biomed. Mater. Res. B 68 (2) (2004) 199-208.

[16] J. Keating, M. Mcqueen, Substitutes for autologous bone graft in orthopaedic trauma, J. Bone Joint Surg. Br. 83 (1) (2001) 3-8.

[17] T.W. Bauer, G.F. Muschler, Bone graft materials: an overview of the basic science, Clin. Orthop. Relat. Res. 371 (2000) 10-27.

[18] S. Bose, M. Roy, A. Bandyopadhyay, Recent advances in bone tissue engineering scaffolds, Trends Biotechnol. 30 (10) (2012) 546-554.

[19] F. Wu, C. Liu, B. O'neill, J. Wei, Y. Ngothai, Fabrication and properties of porous scaffold of magnesium phosphate/polycaprolactone biocomposite for bone tissue engineering, Appl. Surf. Sci. 258 (19) (2012) 7589-7595.

[20] D. Yang, Z. Yang, X. Li, L. Di, H. Zhao, A study of hydroxyapatite/calcium sulphate bioceramics, Ceram. Int. 31 (7) (2005) 1021-1023.

[21] A. Ślósarczyk, J. Czechowska, Z. Paszkiewicz, A. Zima, New bone implant material with calcium sulfate and Ti modified hydroxyapatite, J. Achiev. Mater. Manuf. Eng. 43 (2010) 170-177.

[22] W. Zhou, Y. Xue, X. Ji, G. Yin, N. Zhang, Y. Ren, A novel injectable and degradable calcium phosphate/calcium sulfate bone cement, Afr. J. Biotechnol. 10 (88) (2011) 19449-19457.

[23] J. Ricci, H. Alexander, P. Nadkarni, M. Hawkins, J. Turner, S. Rosenblum, L. Brezenoff, D. De Leonardis, G. Pecora, Biological mechanisms of calcium sulfate replacement by bone, In: Bone Eng, 2000, pp. 332-344.

[24] M. Nilsson, E. Fernandez, S. Sarda, L. Lidgren, J. Planell, Characterization of a novel calcium phosphate/sulphate bone cement, J. Biomed. Mater. Res. 61 (4) (2002) 600-607.

[25] M.A. Rauschmann, T.A. Wichelhaus, V. Stirnal, E. Dingeldein, L. Zichner, R. Schnettler, V. Alt, Nanocrystalline hydroxyapatite and calcium sulphate as biodegradable composite carrier material for local delivery of antibiotics in bone infections, Biomaterials 26 (15) (2005) 2677-2684.

[26] Z. Zhou, E. Cunningham, A. Lennon, H.O. McCarthy, F. Buchanan, S.A. Clarke, N. Dunne, Effects of poly ( $\varepsilon$-caprolactone) coating on the properties of three-dimensional printed porous structures, J. Mech. Behav. Biomed. Mater. 70 (2017) 68-83.

[27] C. Newe, E. Cunningham, F. Buchanan, G. Walker, P. Prendergast, A. Lennon, N. Dunne, Static and dynamic degradation of sintered calcium phosphate ceramics, Key Eng. Mater. 493 (2012) 861-865.

[28] G.N. Bancroft, V.I. Sikavitsas, J. Van Den Dolder, T.L. Sheffield, C.G. Ambrose, J.A Jansen, A.G. Mikos, Fluid flow increases mineralized matrix deposition in 3D perfusion culture of marrow stromal osteoblasts in a dose-dependent manner, Proc. Natl. Acad. Sci. U. S. A. 99 (20) (2002) 12600-12605.

[29] J. Hawkins, M. Cifuentes, N.L. Pleshko, H. Ambia-Sobhan, S.A. Shapses, Energy restriction is associated with lower bone mineral density of the tibia and femur in lean but not obese female rats, J. Nutr. 140 (1) (2010) 31-37.
[30] M. Doube, M.M. Klosowski, I. Arganda-Carreras, F.P. Cordelieres, R.P. Dougherty, J.S. Jackson, B. Schmid, J.R. Hutchinson, S.J. Shefelbine, BoneJ: free and extensible bone image analysis in ImageJ, Bone 47 (6) (2010) 1076-1079.

[31] Z. Zhou, F. Buchanan, C.A. Mitchell, N. Dunne, Effects of heat treatment on the mechanical and degradation properties of 3D-printed calcium-sulphate-based scaffolds, ISRN Biomater. 2013 (2012), (10 Pages).

[32] F.H. Zulkifli, F.S.J. Hussain, M.S.B.A. Rasad, M.M. Yusoff, In vitro degradation study of novel HEC/PVA/collagen nanofibrous scaffold for skin tissue engineering applications, Polym. Degrad. Stab. 110 (2014) 473-481.

[33] H. Shao, Y. He, J. Fu, D. He, X. Yang, J. Xie, C. Yao, J. Ye, S. Xu, Z. Gou, 3D printing magnesium-doped wollastonite/ $\beta$-TCP bioceramics scaffolds with high strength and adjustable degradation, J. Eur. Ceram. Soc. 36 (6) (2016) 1495-1503.

[34] M.T. Fulmer, I.C. Ison, C.R. Hankermayer, B.R. Constantz, J. Ross, Measurements of the solubilities and dissolution rates of several hydroxyapatites, Biomaterials 23 (3) (2002) 751-755.

[35] B. Rai, S. Teoh, D. Hutmacher, T. Cao, K. Ho, Novel PCL-based honeycomb scaffolds as drug delivery systems for rhBMP-2, Biomaterials 26 (17) (2005) 3739-3748.

[36] L.S. Nair, G.T. Laurencin, Biodegradable polymers as biomaterials, Prog. Polym. Sci. 32 (8) (2007) 762-798.

[37] M. Peroglio, L. Gremillard, J. Chevalier, L. Chazeau, C. Gauthier, T. Hamaide, Toughening of bio-ceramics scaffolds by polymer coating, J. Eur. Ceram. Soc. 27 (7) (2007) 2679-2685.

[38] S. Tarafder, S. Bose, Polycaprolactone-coated 3D printed tricalcium phosphate scaffolds for bone tissue engineering: in vitro alendronate release behavior and local delivery effect on in vivo osteogenesis, ACS Appl. Mater. Interfaces 6 (13) (2014) 9955-9965.

[39] Z. Yang, D.A. Yang, H. Zhao, Degradation behavior of calcium sulfate/ $\beta$-tricalcium phosphate composites in tris, Key Eng. Mater. 336 (2007) 1635-1637.

[40] Y. Chang, C.M. Stanford, J.C. Keller, Calcium and phosphate supplementation promotes bone cell mineralization: implications for hydroxyapatite (HA)-enhanced bone formation, J. Biomed. Mater. Res. 52 (2) (2000) 270-278.

[41] A. Ehara, K. Ogata, S. Imazato, S. Ebisu, T. Nakano, Y. Umakoshi, Effects of $\alpha$-TCP and TetCP on MC3T3-E1 proliferation, differentiation and mineralization, Biomaterials 24 (5) (2003) 831-836

[42] M.D. Vlad, E. Şindilar, M.L. Mariñoso, I. Poeată, R. Torres, J. López, M. Barracó, E Fernández, Osteogenic biphasic calcium sulphate dihydrate/iron-modified $\alpha$-tricalcium phosphate bone cement for spinal applications: in vivo study, Acta Biomater. 6 (2) (2010) 607-616.

awkinsifuentesleshko, ) 0

[43] D.R. Carter, W.C. Hayes, Bone compressive strength: the influence of density and strain rate, Science 194 (4270) (1976) 1174-1176.

[44] A.S. Coetzee, Regeneration of bone in the presence of calcium sulfate, Arch. Otolaryngol. Head Neck Surg. 106 (1980) 405.

[45] M. Orsini, G. Orsini, D. Benlloch, J.J. Aranda, P. Lazaro, M. Sanz, M.D. Luca, A Piattelli, Comparison of calcium sulfate and autogenous bone graft to bioabsorbable membranes plus autogenous bone graft in the treatment of intrabony periodontal defects: a split-mouth study, J. Periodontol. 72 (2001) 296-302.

ilssonernandez, J. Biomed. Mater. Res. (

[46] J. Borrelli, W.D. Prickett, W.M. Ricci, Treatment of nonunions and osseous defects with bone graft and calcium sulfate, Clin. Orthop. Relat. Res. 411 (2003) 245-254. 